\title{
RelB acts as a molecular switch driving chronic inflammation in glioblastoma multiforme
}

\author{
Michael R. Waters ${ }^{1}$, Angela S. Gupta ${ }^{1}$, Karli Mockenhaupt ${ }^{1}$, LaShardai N. Brown', Debolina D. Biswas ${ }^{1}$ and \\ Tomasz Kordula ${ }^{1}$
}

\begin{abstract}
Glioblastoma multiforme (GBM) is a primary brain tumor characterized by extensive necrosis and immunosuppressive inflammation. The mechanisms by which this inflammation develops and persists in GBM remain elusive. We identified two cytokines interleukin-1 $\beta$ (IL-1) and oncostatin M (OSM) that strongly negatively correlate with patient survival. We found that these cytokines activate RelB/p50 complexes by a canonical NF-kB pathway, which surprisingly drives expression of proinflammatory cytokines in GBM cells, but leads to their inhibition in non-transformed astrocytes. We discovered that one allele of the gene encoding deacetylase Sirtuin 1 (SIRT1), needed for repression of cytokine genes, is deleted in $80 \%$ of GBM tumors. Furthermore, RelB specifically interacts with a transcription factor Yin Yang 1 (YY1) in GBM cells and activates GBM-specific gene expression programs. As a result, GBM cells continuously secrete proinflammatory cytokines and factors attracting/activating glioma-associated microglia/macrophages and thus, promote a feedforward inflammatory loop.
\end{abstract}

\section{Introduction}

Robust angiogenesis, radioresistance, and invasion make glioblastoma multiforme (GBM) one of the most lethal cancers with patient survival rates that have not improved in decades ${ }^{1,2}$. Although GBM tumors exhibit mutations in known tumor suppressors and oncogenes, they are extensively heterogeneous ${ }^{3}$. Unsupervised clustering analysis of GBM tumors identified four distinct gene expression subtypes, which are now recognized as proneural, neural, classical, and mesenchymal $\mathrm{GBMs}^{4,5}$. These transcriptional subtypes are associated with frequent specific somatic alternations, such as PDGFRA amplifications, and IDH1 and TP53 mutations in the proneural subtype, EGFR alterations in the classical subtype, and NF1 abnormalities in the mesenchymal GBM ${ }^{5,6}$. While inflammation also develops in all GBM subtypes,

Correspondence: Tomasz Kordula (Tomasz.Kordula@vcuhealth.org) 'Department of Biochemistry and Molecular Biology, Virginia Commonwealth, University School of Medicine and the Massey Cancer Center, Richmond, VI 23298, USA extensive necrosis and profound immunosuppressive inflammation characterizes the most common and deadly mesenchymal subtype of GBM, which is more resistant to standard therapies and has the worst prognosis ${ }^{7,8}$. The unique immunosuppressive microenvironment is the major obstacle for immunotherapy, which so far has not been successful in $\mathrm{GBM}^{9-13}$. GBM tumors are extensively infiltrated by immune cells, including glioma-associated microglia/macrophages (GAMs), myeloid-derived suppressor cells, $\mathrm{T}$ cells, and granulocytes ${ }^{14}$. Additionally, expression of cytokines, chemokines, and other inflammatory mediators produced by both GBM cells and GBM microenvironment is most robust in the mesenchymal GBM tumors ${ }^{15}$. The proinflammatory signaling induced by these mediators enhances the proliferation, invasiveness, resistance to apoptosis, maintenance of stem celllike properties, drug resistance of GBM cells, and angiogenesis of GBM tumors, driving tumor progression ${ }^{16,17}$. Despite significant effects on tumor progression, mechanisms by which this immunosuppressive 
inflammation develops in GBM and persists regardless of multiple anti-inflammatory feedback mechanisms remain elusive.

Inflammation is associated with activation of the NF- $\mathrm{kB}$ family of transcription factors (p65, cRel, p105/p50, p100/ $\mathrm{p} 52$, and $\mathrm{RelB}$ ) that regulate a wide-range of processes, including cell survival and both immune and inflammatory responses ${ }^{18}$. Classically, NF-kB proteins are activated either via a canonical or a non-canonical pathway leading to the activation of $\mathrm{p} 65 / \mathrm{p} 50$ or $\mathrm{RelB} / \mathrm{p} 52$ heterodimers, respectively. Although the p65/p50 complexes are activated by many proinflammatory stimuli, the RelB/p52 complexes are activated by a selected group of ligands, and control development of lymphoid organs ${ }^{19}$. It has been proposed that non-canonical pathway-activated RelB/p52 complexes can promote GBM progression ${ }^{20-23}$. However, although aberrant p65/p50 and RelB/ p52 signaling have been linked to oncogenesis and progression $^{24}$, targeting these pathways has not been beneficial $^{24}$, and inhibitors, such as sulfasalazine have failed clinical trials in $\mathrm{GBM}^{25}$. Interestingly, however, RelB can also form RelB/p50/IкB $\alpha$ complexes, activated by canonical stimuli, in cells expressing high levels of RelB ${ }^{26}$. RelB also limits inflammation in innate immune cells and astrocytes by several mechanisms ${ }^{27-29}$. Paradoxically, RelB is also a marker of the highly inflammatory mesenchymal GBM subtype ${ }^{5}$. Given RelB's key role in establishing a negative inflammatory feedback in astrocytes $^{29}$, we asked whether its activation by the canonical NF- $\mathrm{KB}$ pathway regulates inflammation associated with mesenchymal GBM.

\section{Results}

\section{IL-1 and OSM specifically predict short GBM patient survival}

Although proinflammatory cytokines are secreted transiently during acute inflammation, chronic immunosuppressive inflammatory state develops in GBM and promotes tumor progression ${ }^{16,17}$. To identify cytokines which specifically support GBM aggressiveness, we used an unbiased approach and correlated cytokine and cytokine receptor expression with clinical outcome data from The Cancer Genome Atlas (TCGA) (Fig. 1a, b, Supplementary Table 1). We identified two cytokines interleukin-1 $\beta$ (IL-1) and oncostatin M (OSM) (and their receptors) (Fig. 1c) that most strongly negatively correlated with patient survival, in contrast to many others that did not, such as interferon $\beta$ (IFN $\beta$ ) and IFN $\gamma$ (Fig. 1d). Interestingly, expression of IL-1 strongly correlated with expression of OSM (Pearson correlation $=0.769$ ) (Fig. 1e). Remarkably, ranking of every expressed gene in GBM, according to their Pearson correlation, indicated that the $O S M$ gene is the second most correlated with the IL1B gene in the entire genome (Fig. 1f), and patients expressing high levels of both cytokines have very poor prognosis (Fig. 1g). Additionally, IL-1 and OSM are expressed at high levels in mesenchymal GBM (Fig. 2h). Since IL-1 is expressed by GBM tumors ${ }^{30}$, GAMs, microglia, and reactive astrocytes ${ }^{31}$, while OSM is produced only by macrophages and microglia ${ }^{32}$, we hypothesized that chronic elevation of IL-1 and OSM levels initiates programs driving GBM progression.

\section{RelB/p50 canonical signaling in GBM}

Since canonical NF-kB stimuli, such as IL-1, can activate $\mathrm{RelB} / \mathrm{p} 50$ complexes $^{26,29,33}$, we tested whether these complexes are activated by IL-1/OSM in GBM cells. Similarly to what we previously found in astrocytes ${ }^{29,33}$, basal expression of RelB is low in GBM cells. However, IL$1 / \mathrm{OSM}$ induced expression of RelB and p50 in both primary GBM cells and established GBM cell lines (Fig. 2a, b). Similarly to p65 (Supplemewntary Fig. 1a), RelB translocated to the nucleus of the GBM cells in response to IL-1/OSM (Fig. 2c). RelB formed distinctive puncta in the nuclei of GBM cells (Fig. 2d, Supplementary Fig. 1b), and its expression was p65-dependent (Suppementary Fig. 1c). Strikingly, while knockdown of RelB (Supplementary Fig. 2a) enhanced expression of proinflammatory cytokines and COX-2 in primary astrocytes (Supplementary Fig. 2b), it had an opposite effect in primary GBM cells and established GBM cell lines (Fig. 2e). Importantly, IL-1 (alone or together with OSM) induced the formation of RelB/p50 complexes (Fig. 2f). Both RelB and p50, but not p52, bound the target promoters (Fig. 2g). RelB expression was higher in GBM samples than normal brains (Fig. 2h), and RelB was also almost entirely localized in the nuclei in GBM patient samples (Fig. 2i). While previous reports showed that RelB/p52 complexes promote GBM progression $^{20-23}$, we strikingly found that over $80 \%$ of GBMs lost one allele of the NFKB2 (p100/p52), but almost 30\% of GBMs have RelB allele gains (Fig. 2j). In summary, in response to IL-1/OSM, RelB/p50-canonical signaling is activated in GBM cells in vitro, and RelB-canonical signaling is likely more prevalent in GBM in vivo.

\section{Opposing effects of RelB in GBM cells versus astrocytes}

To gain insight into RelB-driven gene expression programs in GBM, we generated U373-RelB-deficient cells using CRISPR/CAS9 (Fig. 3a). Similarly to RelB knockdown (Fig. 2e), knock-out of RelB severely diminished IL1/OSM-induced cytokine expression (Fig. 3b) and also diminished cytokine-induced proliferation (Fig. 3c) and migration (Fig. 3d). In order to define the global role of RelB in GBM cells, we performed RNA-seq analysis of parental and RelB-deficient cells, and conducted differential expression testing and pathway enrichment analysis. RNA-seq analysis indicated that in response to cytokines, RelB overwhelmingly activates genes in GBM, 


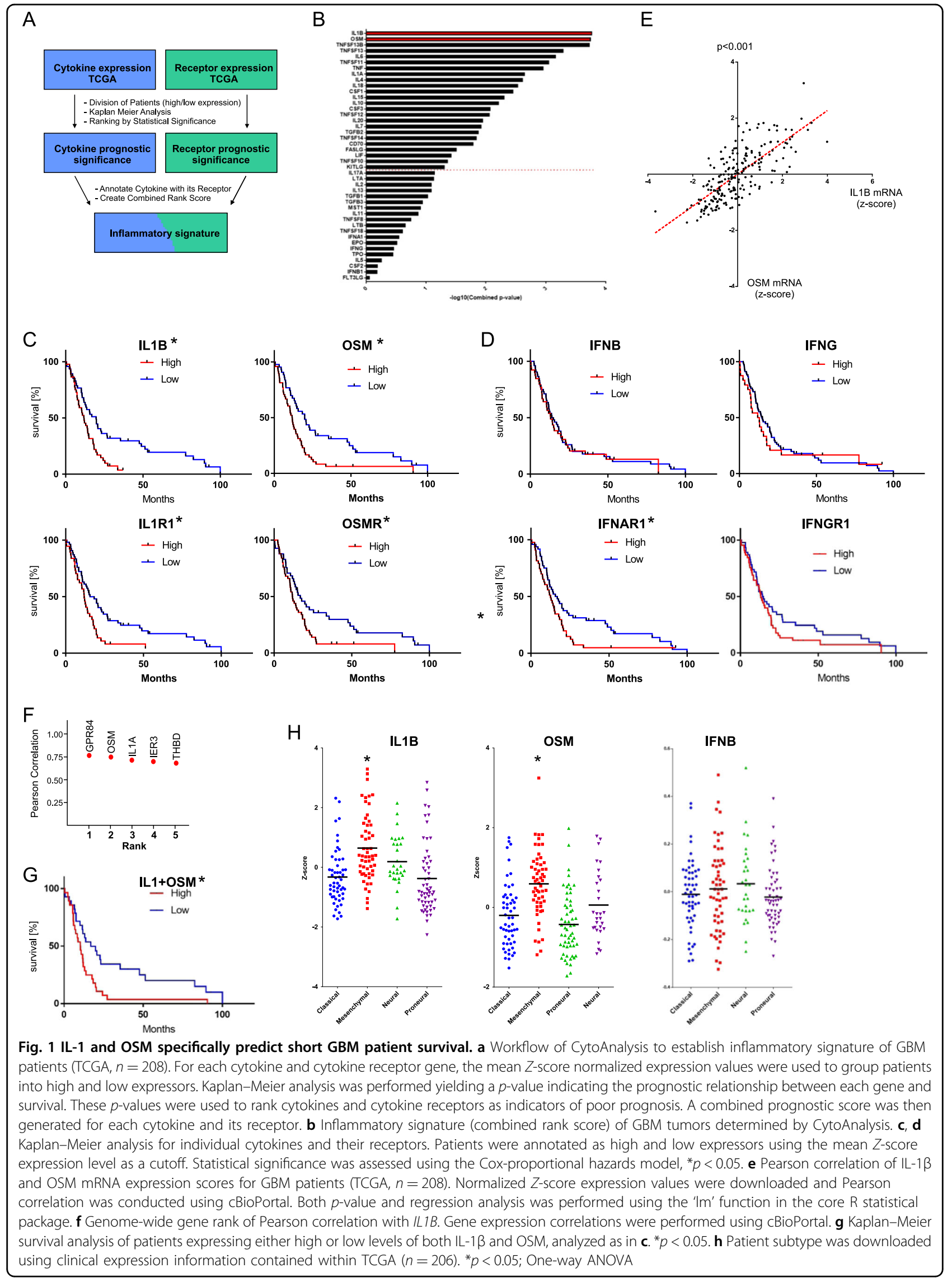




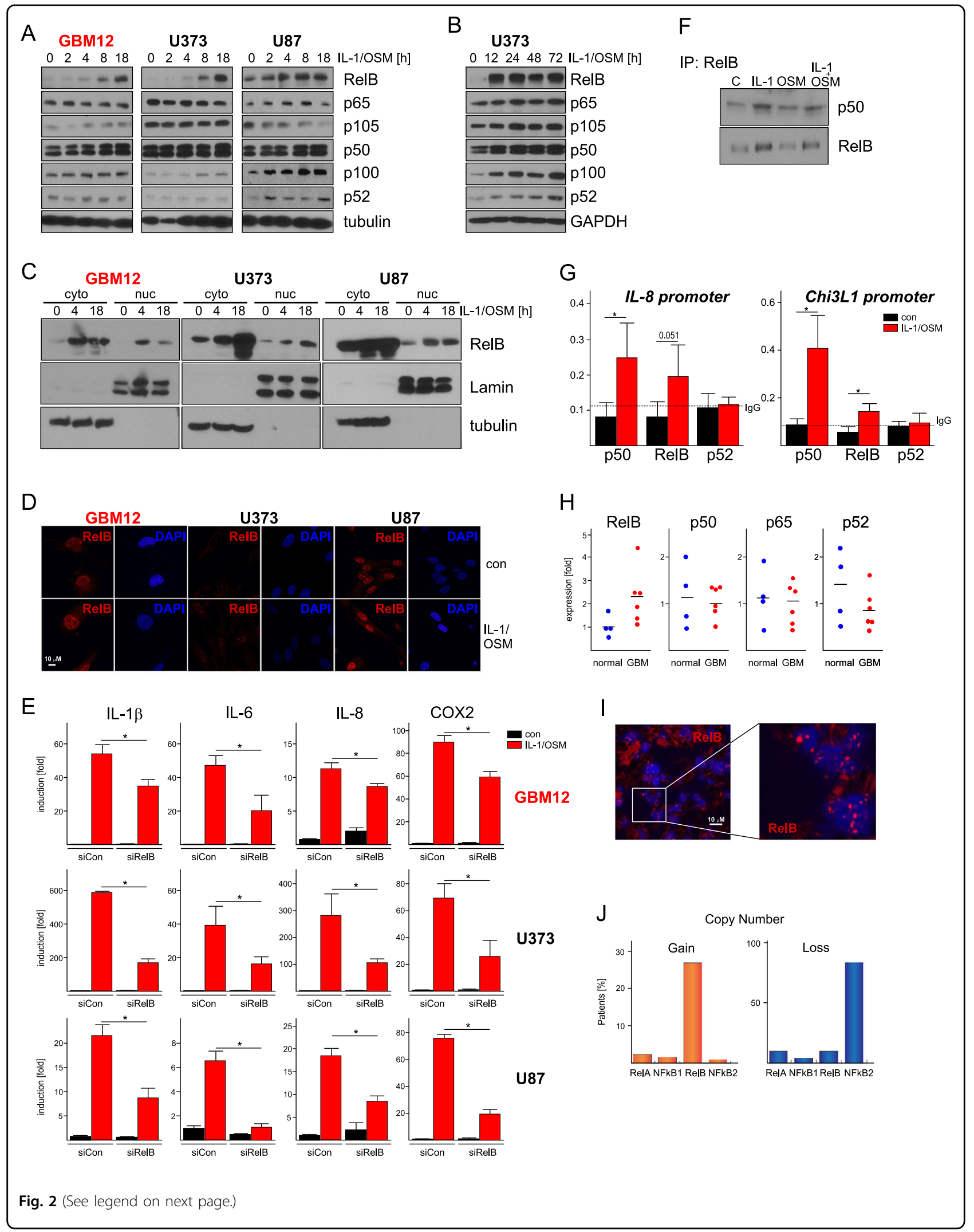


(see figure on previous page)

Fig. 2 IL-1/OSM activate ReIB/p50 in GBM. a Primary GBM12 cells and established U373 and U87 cell lines were stimulated with IL-1/OSM as indicated. Expression of RelB, p65, p105, p50, p100, and p52 was analyzed by western blotting. Tubulin was used as a loading control. b U373 cells were treated with IL-1/OSM as indicated, expression was assessed by western blotting. c Nuclear and cytosolic fractions were prepared from control or IL-1/OSM-stimulated cells (at indicated time points), and RelB expression was analyzed by western blotting. Lamin A/C and tubulin were used to examine purity of the fractions. $\mathbf{d}$ Cells were stimulated for $18 \mathrm{~h}$, and RelB visualized by immunofluorescence. DAPI was used to stain nuclei. e Cells transfected with the indicated siRNAs were stimulated $48 \mathrm{~h}$ later with IL-1/OSM for $18 \mathrm{~h}$. Expression was analyzed by qPCR (three experiments, error bars represent s.d., ${ }^{*} p<0.05$ (two-way ANOVA, Sidak's test). $\mathbf{f} \mathrm{U} 373$ were stimulated for $8 \mathrm{~h}$, RelB was immunoprecipitated, and p50 and RelB were detected by western blotting. $\mathbf{g}$ Binding of RelB, p50, and p52 at the indicated cytokine promoters was analyzed by ChIP. U373 cells were stimulated with IL-1/OSM for $8 \mathrm{~h}$. Normalized binding is shown. IgG was used as a control for IP (dotted line). $n=3-6$, error bars represent s.d.., ${ }^{*} p<0.05$ (T-test, Sidak's test). $\mathbf{h}$ Expression was analyzed by qPCR in human GBM tumors $(n=6)$ and normal brains $(n=4)$. i RelB was visualized in tumor sample of GBM patient by immunofluorescence. DAPI was used to counterstain nuclei. $\mathbf{j}$ Genomic copy number analysis of NF-kB family members, GISTIC data downloaded from TCGA via cBioPortal $(n=206)$

and the enriched pathways are involved in proinflammatory responses $\left(p=1.37 \times 10^{-7}\right)$, perpetuating inflammation, and inflammatory cell chemotaxis (Fig. 3e). Conversely, using the RelB knock-down approach, we found that RelB inhibits proinflammatory response $(p=$ $1.92 \times 10^{-10}$ ) in primary astrocytes (Fig. 3e). Thus, this genome-wide expression analysis suggests that RelB functions mostly as a transcriptional repressor in astrocytes, but as a transcriptional activator in GBM cells in vitro. To test whether RelB functions as a transcriptional activator in GBM in vivo, we performed differential expression testing and pathway enrichment analysis of patients expressing either high or low levels of RelB. Interestingly, patients expressing high RelB levels show enrichment of the same pathways (Fig. 3f), which were activated by RelB in GBM cells in vitro (Fig. 3e).

\section{RelB coordinates recruitment and activation of myeloid cells in GBM}

To determine which of the RelB-dependent genes identified by RNA-seq have the greatest impact on patient survival, we wrote an in house R-script to perform "iterative Kaplan-Meier analysis" (Fig. 4a). Importantly, the vast majority of RelB-dependent genes, statistically important for patient prognosis, are markers of poor prognosis (Fig. 4b). Pathway enrichment analysis for these genes showed that the inflammatory genes including those chemotactic for myeloid cells are the most overrepresented in patients expressing high levels of RelB (Fig. 4c). These data suggested that a major effect of RelB programs in GBM may be recruitment of myeloid cells, which leads to more aggressive tumors $^{34}$. Although RelB did not induce classical drivers of the M1 (IFN $y$ and TNF $\alpha$ ) or the M2 phenotype (IL-4, IL13), prognostically important genes specifically induced by RelB are CSF1, CSF2, CSF3, CCL2, CCL7, CXCL2, and CXCL3 (Fig. 4d). Since proteins encoded by these genes are known to activate and attract myeloid cells to the sites of inflammation, RelB may be controlling recruitment and activation of GAMs.

\section{Loss of SIRT1 is associated with persistent inflammation in GBM}

$\mathrm{NAD}^{+}$-dependent histone deacetylase SIRT1 has been implicated in RelB-mediated epigenetic silencing that regulates LPS tolerance in macrophages ${ }^{35,36}$. SIRT1 also regulates adaptive responses of astrocytes by suppressing IL-1-induced activation of cytokine genes ${ }^{29}$. Although SIRT1 suppressed IL-1/OSM-induced cytokine expression in astrocytes (Fig. 5a), it surprisingly had no effect in GBM cells (Supplementary Fig. 3a). Mining of TCGA database showed that one allele of the SIRT1 gene is deleted in $\sim 80 \%$ of GBM tumors (Fig. $5 \mathrm{~b}$ ). We also found lower expression of SIRT1 mRNA in GBMs than normal (matching) brain (Fig. 5c), and confirmed these patient's samples on the protein level by IHC (Fig. 5d). These findings were further confirmed in vitro since SIRT1 mRNA levels (Fig. 5e), SIRT1 protein (Fig. 5f), and SIRT1 activity (Fig. 5g) were decreased in GBM cell lines and primary GBM cells in comparison to astrocytes. Remarkably, loss of one allele of the SIRT1 gene leads to poor patient survival (Fig. 5h) (disease-free survival is 4.9 months in comparison to 22 months for diploid patients). We addressed the importance of SIRT1 in RelBmediated regulation by overexpression of SIRT1 in GBM cells, which significantly diminished expression of IL-6 and IL-8 but had no effect on IL-1 (Fig. 5i). Importantly, the effect of SIRT1 was RelB-dependent, since SIRT1 overexpression was not effective in the absence of RelB (Fig. 5j). Overexpression of SIRT1 also decreased rate of glycolysis in GBM cells (Supplementary Fig. 3b). Although SIRT1 can deacetylate histones, acetylation of histones was not diminished at the cytokine promoters in response to IL1/OSM in GBM cells (Fig. 5k). SIRT1 was also absent at these promoters even though they were active, as indicated by tri-methylation of histone $\mathrm{H} 3$ at lysine 4 (Fig. 5k). Significantly, patients expressing RelB at high levels but SIRT1 at low levels have very poor survival prognosis (Fig. 5l). These data suggest that although SIRT1 represses cytokine genes in astrocytes, RelB/SIRT1-dependent 


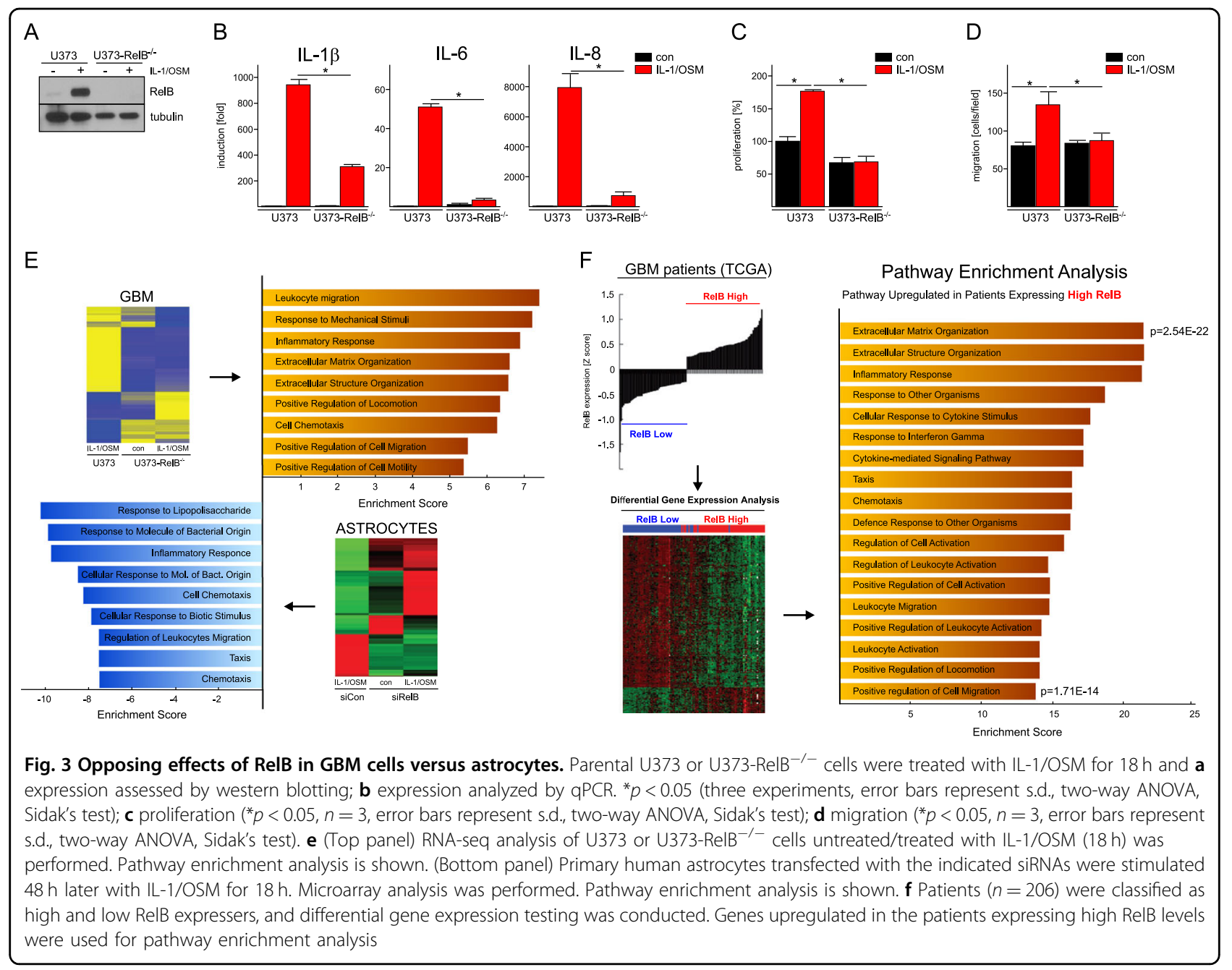

repression does not function in GBM cells due to lower expression/activity of SIRT1.

\section{Yin Yang 1 (YY1) specifically upregulates cytokine expression in GBM cells}

Although lower expression/activity of SIRT1 could explain lack of cytokine gene silencing by RelB in GBM cells, it could not explain "aberrant" RelB-dependent activation of cytokine expression in these cells. We hypothesized that this activation may depend on an additional transcription factor that affects RelB activity in GBM cells but not astrocytes. To identify this factor, we analyzed $2.5 \mathrm{~kb}$-long promoter regions of the RelBdependent genes for the presence of regulatory elements using the EnrichR algorithm (Fig. 6a) ${ }^{37}$. We identified several regulatory elements, including a motif that binds YY1, a GLI-Krüppel-related zinc-finger transcription factor regulating formation of enhancer-promoter loops, recruitment of corepressors and coactivators, and thus shaping chromatin structure ${ }^{38}$. Indeed, analysis of publicly available ChIP-seq datasets (Fig. 6b) indicated presence of overlapping peaks for both RelB and YY1 at multiple genomic locations, including the $I L 1 B$ gene (Fig. 6c). To test whether YY1 differentially functions in GBM cells versus astrocytes, we depleted YY1 in these cells. We found that although cytokine expression is YY1independent in astrocytes, it is upregulated by YY1 in GBM cells (Fig. 6d). Significantly, we found that YY1 is localized almost exclusively to cytoplasm of astrocytes, whereas it is almost entirely nuclear in GBM cells (Fig. 6e, f, Supplementary Fig. 4a), which directly correlates with YY1-dependent regulation of cytokine expression. YY1 was also present in the nuclei of GBM cells in tumors, while its localization was mostly cytoplasmic in nearby normal tissue (Fig. 6g). Since YY1 can form complexes with RelB and Oct- $2^{39}$, and Oct- 2 elements are the second most enriched motifs in RelB-regulated genes (POU2F2, Fig. 6a), we tested whether YY1 interacts with RelB and p50 in GBM cells. We could co-IP YY1 with RelB (Fig. 6h), and p50, but not p105 (Fig. 6i). YY1 also colocalized with RelB in the nuclei of GBM cells (Fig. 6f). Furthermore, knock-down of YY1 together with RelB 


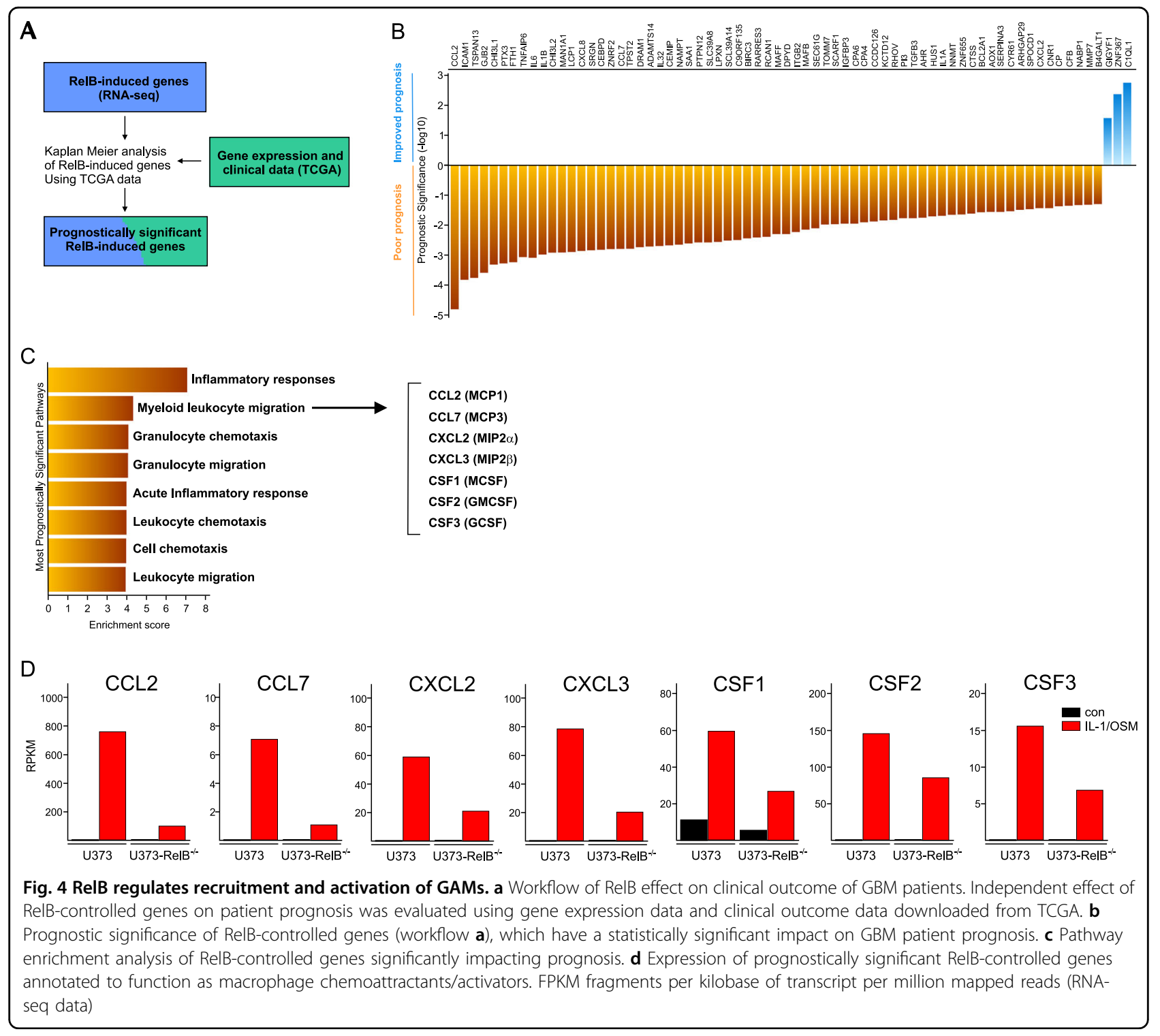

(Supplementary Fig. 4b) did not have an additive effect on cytokine expression (Fig. 6j), suggesting that they function together. YY1 was also bound to the cytokine promoters in GBM cells (Fig. 6k). We concluded that nuclear YY1 is likely responsible for specific upregulation of cytokine expression by RelB in GBM cells.

\section{Discussion}

Various components of the non-canonical NF-kB-signaling pathway, including TWEAK, cIAP $1 / 2$, NIK, and RelB, have been implicated in tumorigenesis ${ }^{40}$, and these proteins also promote glioma cell invasion ${ }^{20-23,41}$. In contrast to RelB/p52 complexes, the less understood RelB/p50 complexes are activated by the canonical pathway, limit inflammation in innate immune cells, control adaptive responses in astrocytes, are not easily removed from DNA, and provide long-lasting effects ${ }^{19,29,36}$, but they have not been shown to play any role in GBM. Our data indicate that the inflammatory milieu of GBM is rich in cytokines that are known to activate RelB/p50 complexes in normal cells ${ }^{33}$. We further show that RelB/p50 complexes are also activated in GBM cells, suggesting an additional previously unidentified mechanism by which RelB affects GBM biology and patient survival. The importance of the canonical RelB/p50 signaling is further supported by a significant increase in genomic dose of the $R E L B$ gene (30\% of GBM patients) but striking $80 \%$ loss of the NFKB2 (encoding $\mathrm{p} 100 / \mathrm{p} 52$ ). These data strongly support the idea that p52-independent RelB signaling is critical in GBM development or progression.

Our previous ${ }^{29}$ and current data show that cytokineinduced RelB/p50 complexes suppress expression of the 


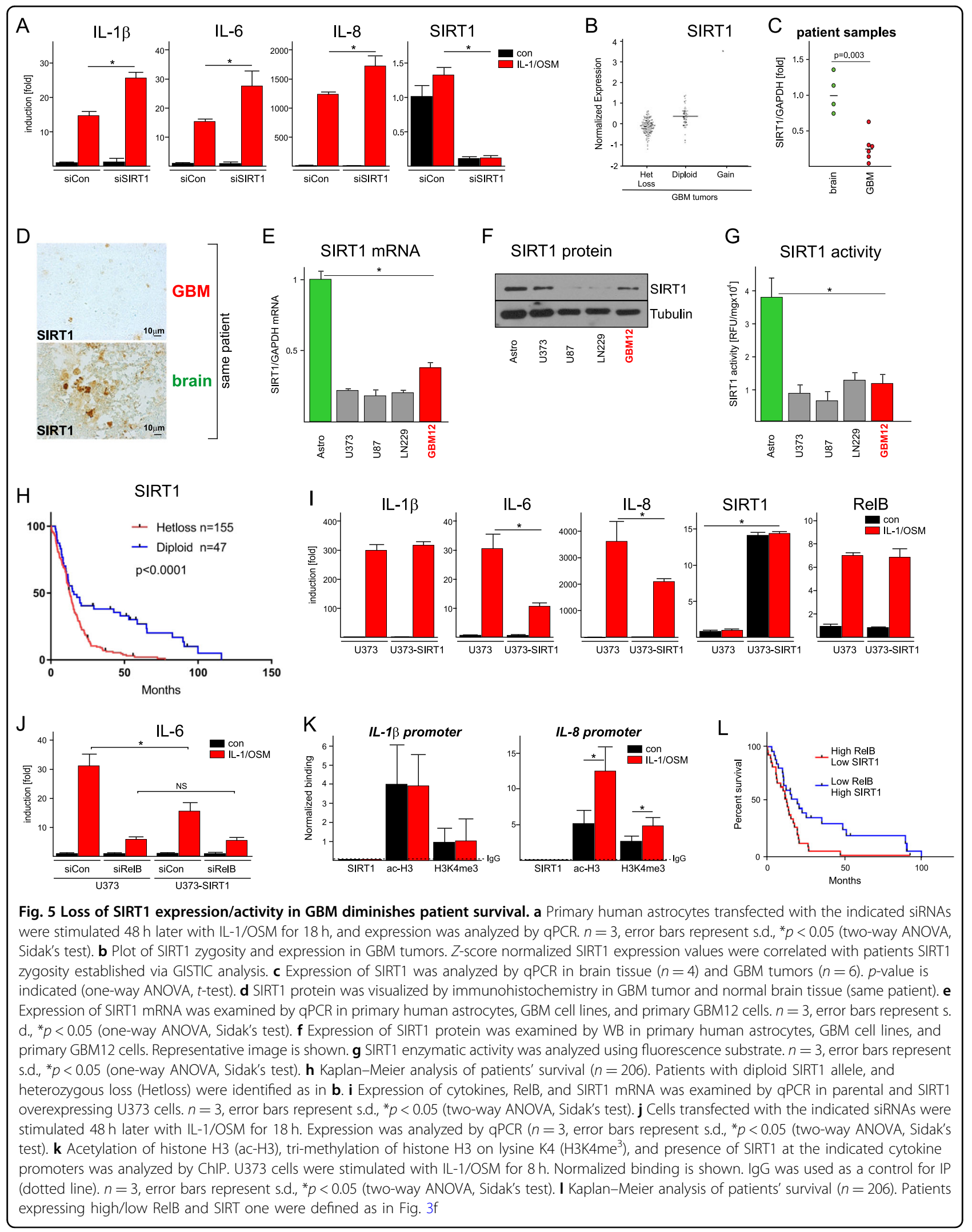




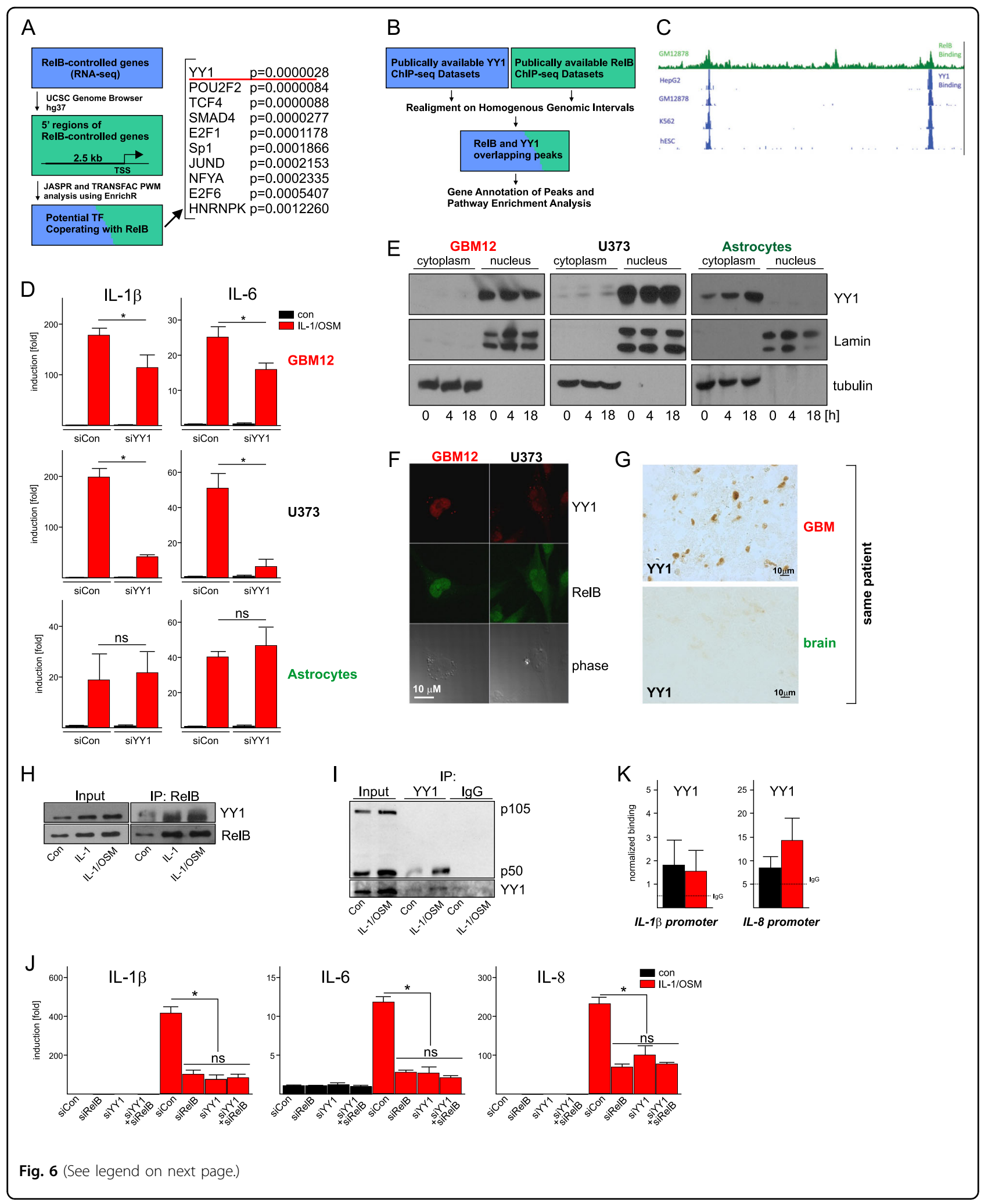


(see figure on previous page)

Fig. 6 YY1 upregulates cytokine expression in GBM but not astrocytes. a Workflow for the identification of putative transcription factors cooperating with RelB (left panel). Rank list of regulatory elements in RelB-controlled genes. $p$-values were directly generated by PWM. b Workflow for YY1 and RelB ChIPseq data processing. c Representative RelB/YY1 peaks. d Cells transfected with the indicated siRNAs were stimulated $48 \mathrm{~h}$ later with IL-1/OSM for 18 h. Expression was analyzed by qPCR. $n=3$, error bars represent s.d., ${ }^{*} p<0.05$ (two-way ANOVA, Sidak's test). e Nuclear and cytosolic fractions were prepared from control or IL-1/OSM-stimulated cells (at indicated time points), and YY1 expression was analyzed by western blotting. Lamin A/C and tubulin were used to examine purity of the fractions. $\mathbf{f}$ Cells were stimulated for $18 \mathrm{~h}$, and $\mathrm{YY} 1$ and RelB visualized by

immunofluorescence. $\mathbf{g}$ YY1 protein was visualized by immunohistochemistry in GBM tumor and normal brain tissue (same patient). $\mathbf{h}$, i U373 cells were stimulated with IL-1 and/or OSM for $18 \mathrm{~h}$. $\mathbf{h}$ RelB was immunoprecipitated. RelB and YY1 were detected in immunoprecipitates by western blotting. $\mathbf{i}$ YY1 was immunoprecipitated. p50, p105, and YY1 were detected in immunoprecipitates by western blotting. Expression in the lysates is shown (Input). $\mathbf{j}$ Cells transfected with the indicated siRNAs were stimulated $48 \mathrm{~h}$ later with IL-1/OSM for $18 \mathrm{~h}$. Expression was analyzed by qPCR. $n=$ 3, error bars represent s.d., ${ }^{*} p<0.05$ (two-way ANOVA, Sidak's test). $\mathbf{k}$ YY1 binding at the cytokine promoters was analyzed by ChIP. U373 cells were stimulated with IL-1/OSM for $8 \mathrm{~h}$. Normalized binding is shown. IgG was used as a control for IP (dotted line). $n=3$

cytokine genes in astrocytes. This is in agreement with the silencing role of RelB in macrophages ${ }^{36}$ and microglia ${ }^{42}$. While silencing of the cytokine genes in macrophages and astrocytes depends on RelB and SIRT1 ${ }^{36,43}$, proposed repressive mechanisms are different with the role of SIRT1 being elusive in astrocytes. In contrast, we show that RelB supports cytokine gene expression in GBM cells, while SIRT1 has no effect. Although we found astonishingly frequent loss of one allele of the SIRT1 gene in GBM tumors and diminished expression of SIRT1 in GBM cells, the activity of SIRT1 may be further diminished by high redox status of GBM cells ${ }^{44}$ or by post-translational modifications of SIRT $1^{45}$. These mechanisms are all likely responsible for the diminished activity of SIRT1 in GBM tumors and cells.

Activation of the cytokine genes by RelB in GBM cells is the surprising result indicating that RelB acts as a molecular switch converting RelB-dependent silencing into transcriptional activation fueling immunosuppressive inflammation. We identified YY1 as a unique modifier of RelB-dependent functions in GBM cells. Although YY1 forms complexes with RelB that may directly affect RelB functions, it is also possible that YY1 cooperates with RelB by forming active chromatin loops ${ }^{38}$. However, functions of YY1 are restricted to actively proliferating cells since YY1 is sequestered to the cytoplasm of non-dividing cells by Retinoblastoma protein ${ }^{46}$. Interestingly, YY1 has also been linked to oncogenesis, and its targeting may be beneficial $^{47}$. YY1 has opposing effects to $\mathrm{p} 65 / \mathrm{p} 50$ on gene expression $^{48}$, and its expression can be induced by $\operatorname{RelB}^{49}$. It remains to be established whether both RelB and YY1 affect tumor generation or progression, but the later seems to be more likely based on our data.

Our unbiased approach identified prognostically important RelB-dependent genes in GBM. In general, RelB supports multiple aspects of inflammation, including cytokine production by GBM cells. However, we speculate that the main outcome of the RelB-controlled program in GBM is the recruitment and activation of GAMs into tumors, which may be critical since increased myeloid cell infiltration is a marker of aggressive disease. GAMs were originally proposed to have an M2 signature ${ }^{50}$, but recently they have been shown to resemble the undifferentiated but active M0 phenotype ${ }^{51}$. Although GBM cells do not secrete classical drivers of the M1 or M2 phenotypes (IFN $\gamma$, TNF $\alpha$, IL-4, and IL-13), RelB in GBM cells supports expression of known activators and chemoattractants of GAMs, including members of the macrophage inflammatory protein (MIP), colony-stimulating factor (CSF), and the macrophage chemotactic protein (MCP) families ${ }^{50,52}$.

In conclusion, we propose that although RelB coordinates anti-inflammatory feedback in astrocytes ${ }^{29}$, this mechanism does not function in GBM cells due to both the limited activity of SIRT1 and the presence of YY1 in the nuclei. As a result, GBM cells continuously secrete cytokines and factors attracting/activating GAMs, and thus promote a feedforward immunosuppressive inflammatory loop. Our studies provide a paradigm shift on the role of RelB in GBM. The RelB/p50 complex may emerge as a new target for future interventions to control GBM, and likely other malignances associated with RelBdependent chronic inflammation.

\section{Matherials and methods \\ Cell culture and stimulation}

Human cortical astrocyte cultures were established using cerebral tissue provided by Advanced Bioscience Resources, and the protocol for obtaining postmortem fetal neural tissue complied with the federal guidelines for fetal research and with the Uniformed Anatomical Gift Act. Astrocytes were cultured as described previously ${ }^{33,53}$. Human glioblastoma U373-MG cells were obtained from American Type Culture Collection, whereas human glioma U87 cells were obtained from Dr. Jaharul Haque (Cleveland Clinic Foundation). Primary GBM12 cells were obtained from Dr. Paul Dent (Virginia Commonwealth University). Cells were cultured in DMEM supplemented 
with $10 \%$ FBS, antibiotics, sodium pyruvate, and non-essential amino acids. Cells were stimulated with $25 \mathrm{ng} / \mathrm{ml}$ OSM (R\&D Systems) and $10 \mathrm{ng} / \mathrm{ml} \mathrm{IL-1 \beta}$ (Peprotech).

\section{Knockdown}

Expression was down-regulated using SmartPool siRNAs transfected with Dharmafect 1 (Dharmacon), according to the manufacturer's instructions. The following sequences were targeted: (RelB; CAUCAGAGCU GCGGAUUUG, GCCCGUCUAUGACAAGAAA, GCAC AGAUGAAUUGGAGAU, and GUACCUGCCUCGCGA CCAU), (SIRT1; GUACAAACUUCUAGGAAUG, GU AGGCGGCUUGAUGGUAA, GCGAUUGGGUACCGA GAUA, and GGAUAG GUCCAUAUACUUU).

\section{Quantitative qPCR}

RNA was isolated using Trizol (Invitrogen) and $1 \mu \mathrm{g}$ was reverse-transcribed using the High Capacity cDNA Archive kit (Applied Biosystems). Expression levels were determined using primer-probe sets and TaqMan Universal PCR Master Mix (Applied Biosystems). The cDNAs were diluted 10-fold (target genes) or 100-fold (GAPDH). Gene expression levels were normalized to GAPDH mRNA levels, and presented as a fold induction.

\section{Western blotting}

The cells were lysed in $10 \mathrm{mM}$ Tris (pH 7.4), $150 \mathrm{mM}$ sodium chloride, $1 \mathrm{mM}$ EDTA, $0.5 \%$ Nonidet P-40, $1 \%$ Triton X-100, $1 \mathrm{mM}$ sodium orthovanadate, $0.2 \mathrm{mM}$ PMSF, and protease inhibitor mixture (Roche Applied Science). Samples were separated using SDS-PAGE and transferred onto nitrocellulose membranes. The anti$\beta$-tubulin (sc-9104), anti-RelB (sc-226), anti-p65 (sc-372), anti-p105/p50 (sc-8414), anti-IkB $\alpha$ (sc-371), and antiSIRT1 (sc-15404) antibodies (Santa Cruz Biotechnology); anti-Lamin A/C (2032), anti-myc (2276), and anti-p52 (4882) (Cell Signaling); anti-YY1 (A302-779A) (Bethyl Laboratories), and anti-flag (F1804) antibodies (SigmaAldrich) were used. Antigen-antibody complexes were visualized by ECL using Immobilon Western blotting kit (Millipore).

\section{Immunoprecipitation}

Two hundred to three hundred micrograms of protein lysates, were pre-cleared with $10 \mu \mathrm{l}$ of the protein GSepharose beads (GE Healthcare) for $1 \mathrm{~h}$. The lysates were incubated with antibodies overnight at $4{ }^{\circ} \mathrm{C}$, and then with $25 \mu \mathrm{l}$ protein G-Sepharose beads for $2 \mathrm{~h}$ at $4{ }^{\circ} \mathrm{C}$. The beads were washed with the lysis buffer, and proteins eluted in sample buffer. Flag-tagged RelB was immunoprecipitated with anti-Flag-M2 beads and eluted with Flag peptide (Sigma).

\section{Generation of SIRT1 overexpressing cells}

To generate stable clones, $4 \mu \mathrm{g}$ SIRT1 expression plasmid were transfected into U373 cells. Clones selected in DMEM containing $0.4 \mu \mathrm{g} / \mathrm{ml}$ G418 were subsequently pooled.

\section{Glycolysis assay}

Glycolysis in cultured cells was measured exactly as described before ${ }^{54}$. The conversion of D- $\left[5^{3} \mathrm{H}(\mathrm{N})\right]$-glucose to ${ }^{3} \mathrm{H}_{2} \mathrm{O}$ was calculated, and expressed as glycolytic rate (\% glucose conversion $/ 10^{6}$ cells $/ 6 \mathrm{~h}$ ).

\section{Unbiased cytokine Kaplan-Meier analysis}

Cytokine and Cytokine Receptor $Z$-score normalized expression data was downloaded from TCGA ${ }^{55}$. Patients were divided into high and low expressing groups based on the mean value of gene expression. Kaplan-Meier analysis was performed for each gene based on high and low expression groups. Cytokines/receptors were ranked based on the statistical significance of the individual Kaplan-Meier analysis. Rank of cytokines and cytokine receptors were combined to create a combined rank score to indicate the prognostic impact of each cytokine signaling program.

\section{Fractionation}

Cells were washed with cold PBS and re-suspended in buffer containing $10 \mathrm{mM}$ Hepes $(\mathrm{pH} 7.8), 10 \mathrm{mM} \mathrm{KCl}$, $0.1 \mathrm{mM}$ EDTA, $1 \mathrm{mM}$ sodium orthovanadate, $1 \mathrm{mM}$ DTT, 1:500 protease inhibitors (Sigma), and 0.2 mM PMSF, and incubated on ice for $15 \mathrm{~min}$. NP-40 was added (to $0.75 \%$ ) and cells were vortexed for $10 \mathrm{~s}$. Nuclei and cytoplasm were separated by centrifugation at $3000 \mathrm{rpm}$ for $3 \mathrm{~min}$ at $4{ }^{\circ} \mathrm{C}$. Nuclei were re-suspended in buffer containing $20 \mathrm{mM}$ Hepes (pH 7.8), 0.4 M NaCl, $1 \mathrm{mM}$ EDTA, $1 \mathrm{mM}$ sodium orthovanadate, $1 \mathrm{mM}$ DTT, and protease inhibitor coctail and incubated on ice for $15 \mathrm{~min}$. Nuclear extracts were cleared by centrifugation at $14,000 \times g$ for 5 min at $4{ }^{\circ} \mathrm{C}$.

\section{Generation of U373-RelB ${ }^{-1-}$ cells}

Guide RNAs (5'-caccgGGTCTGGCGACGCGGCGAC $\mathrm{T}-3^{\prime}$ and $5^{\prime}$-aaacAGTCGCCGCGTCGCCAGACCc- $3^{\prime}$ ) targeting RelB gene were designed using the MIT CRISPR guide design tool (http://crispr.mit.edu/). Guide RNA was cloned into BsmBI-digested LentiCRISPRV2 (Addgene). Viral particles were packaged in HEK293T cells using standard approaches. U373 cells were infected, selected in medium containing $75 \mu \mathrm{g} / \mathrm{ml}$ puromycin, and individual colonies were isolated and screened for homozygous RelB knockout. The absence of the off-targets was verified by PCR amplification of predicted off-target fragments and sequencing. 


\section{Migration}

Cells were cultured in six-well plates and serum starved overnight. Cells were stimulated, wounded, and pictures were taken at time 0 and $24 \mathrm{~h}$. Cells migrating past original wound boundary were enumerated and reported.

\section{Proliferation}

Proliferation was examined using WST-8 Cell Proliferation Assay (Dojindo Molecular Technologies).

\section{SIRT1 activity assay}

SIRT1 activities were determined using fluorometric kit (Abcam). Cells were lysed in lysis buffer and then sonicated on ice. $200 \mu \mathrm{g}$ of cell lysate was incubated with antiSIRT1 antibodies overnight at $4{ }^{\circ} \mathrm{C}$ and then with $20 \mu \mathrm{l}$ of $50 \%$ Protein A beads for $3 \mathrm{~h}$ at $4{ }^{\circ} \mathrm{C}$. Beads were washed in SIRT1 assay buffer (50 mM Tris pH 8.8, $0.5 \mathrm{mM}$ DTT). Activity of immunoprecipitated SIRT1 was determined using flouro-substrate mixture according to instructions, and absorbance at $455 \mathrm{~nm}$ was tabulated.

\section{Clinical samples}

Both patient tissue and RNA samples were provided by the VCU Tissue Acquisition and Analysis core.

\section{Immunoflouresence}

Cells cultured on coverslips were washed with PBS and fixed in $2.5 \%$ paraformaldehyde for $10 \mathrm{~min}$ at room temperature. Cells were washed with $0.3 \mathrm{M}$ glycine and permeabilized with $0.1 \%$ Triton X-100. Coverslips were blocked with $5 \%$ BSA/1\% normal goat serum for $1 \mathrm{~h}$, incubated with primary antibodies overnight at $4{ }^{\circ} \mathrm{C}$, washed with PBS and incubated with secondary antibodies for $1 \mathrm{~h}$. Coverslips were washed again, counterstained with Hoescht and mounted using VectaShield (Vector Laboratories).

\section{Immunohistochemistry}

Slides were fixed in ice cold acetone for $20 \mathrm{~min}$, incubated in $1 \%$ hydrogen peroxide, washed in PBS, and blocked in $5 \%$ BSA/1\% normal goat serum for $1 \mathrm{~h}$. The slides were then incubated with primary antibodies in blocking buffer overnight at $4{ }^{\circ} \mathrm{C}$. Slides were subsequently washed in PBS and incubated with EnVision ${ }^{+}$secondary reagent (Agilent) for $20 \mathrm{~min}$, washed in $\mathrm{PBS}$, and then exposed using $\mathrm{DAB}^{+}$chromogen (Agilent) for $10 \mathrm{~min}$.

\section{Microarray processing and differential expression analysis}

Microarrays were processed using the $R$ statistical package requiring the libraries 'affy', 'affyPLM'. Quality control was performed using analysis of $3^{\prime} / 5^{\prime}$ ratios, generating representative array images, and creating RNA degradation plots after generating affybatch objects using the 'affy' and 'affPLM' libraries. Expression summaries of arrays were generated after background correction, and normalization using robust multichip averaging. Statistical significance was assessed using S-testing.

\section{RNA-seq processing and differential expression analysis}

RNA was isolated using the 'mirVana RNA isolation kit' (ThermoFisher Scientific). RNA was sent to the University of Cincinnati Genomics Core for quality control and RNA-sequencing analysis. Data was analyzed using the tuxedo pathway, fastq read files were aligned using BowTie2, transcript assembly, and FPKM estimates achieved using CuffLinks, and testing for differential gene expression and promoter usage were accomplished using CuffDff.

\section{ChIP assay}

The cells were cross-linked with $1 \%$ formaldehyde for $10 \mathrm{~min}$ at $37^{\circ} \mathrm{C}$ and washed with ice-cold PBS containing $125 \mathrm{mM}$ glycine. Chromatin was sheared using a Diagenode Bioruptor (Liège) on high setting for two $10 \mathrm{~min}$ intervals (30 s on/off). Anti-SIRT1, anti-YY1 (both Santa Cruz Biotechnology), and anti-acH3 (Millipore) antibodies were used. DNA was detected by qPCR using TaqMan primers: IL1B forward: $5^{\prime}$-aatttaaaacattcttctaa cgtggg-3', reverse: 5'-ggagtagcaaactatgacacattttg- $3^{\prime}$, and probe: $5^{\prime}$-[6-FAM] caactgcacaacgattgtcaggaaaa[BHQ1aQ]-3'; IL8 forward: 5'-gtgcataagttctctagtagggtgatg-3', reverse: $5^{\prime}$-ggctcttgtcctagaagcttgtgt- $3^{\prime}$, and probe: $5^{\prime}$-[6FAM] cactccataaggcacaaactttcagag[BHQ1a-Q]-3'; Chi3L1 forward: $5^{\prime}$-gtgcagccgcccota- $3^{\prime}$, reverse: $5^{\prime}$-gcaatttacatgctgattatttagaggg- $3^{\prime}$, and probe: $5^{\prime}$-[6-FAM]gcaaaatagcaccggggcttaaag[BHQ1a-Q]-3'. qPCR data was calculated as percent input; IgG was used as a control.

\section{Pearson correlation and gene copy number analysis}

Genome-wide analysis of Pearson correlation (with IL$1 \beta$ expression) and Identification of Copy Number Variation was conducted using the co-expression functionality and GISTIC, respectively, for the data downloaded from the CBioPortal to TCGA ${ }^{55}$.

\section{Patient stratification, differential gene expression, pathway} enrichment, and prognostic significance analyses

$Z$-score normalized RelB expression data was downloaded from TCGA via cBioPortal $(n=206)$. Patients with $Z$-score of $>0.25$ or $<-0.25$ were classified as high and low expressors. Differential gene expression testing was conducted using the 'limma' library of the $\mathrm{R}$ statistical package. Genes upregulated in patients expressing high RelB levels were used for pathway enrichment analysis using the EnrichR software package ${ }^{37}$. Prognostic significance was established via Kaplan-Meier analysis, comparing patients expressing RelB above of or below the mean level of gene expression. 


\section{Promoter analysis and identification of YY1}

$2.5 \mathrm{~kb}-$ long regions surrounding the transcription start sites of the RelB-controlled genes were downloaded from the hg37 genome using the UCSC browser, and analyzed for the presence of regulatory elements (JASPAR and TRANSFAC databases) using the 'PathView' and 'EnrichR' R-packages (RelB-controlled vs. a random genes).

\section{CytoAnalysis}

CIBERSORT analysis was conducted using default settings as described ${ }^{56}$.

\section{Statistical analysis}

All experiments were repeated at least three times with consistent results. Data are presented as mean \pm SD. Statistical analysis was performed using GraphPad Prism 7. Values are displayed as mean \pm standard deviation. $T$-tests and ANOVAs were performed as indicated. Sidak's or Tukey's test was performed to compare multiple groups.

\section{Acknowledgements}

This work was supported by NIH R21NS102802 and VCU PeRQ grants (to T.K.). M.R.W. and A.S.G. were supported by the F30CA203447 and F30CA200252 fellowships from the NIH. Microscopy was performed at the VCU Microscopy Facility supported, in part, by NIH P30CA016059. We would like to thank Dr. Yibao Ma for help with glycolysis assays.

\section{Conflict of interest}

The authors declare that they have no conflict of interest.

\section{Publisher's note}

Springer Nature remains neutral with regard to jurisdictional claims in published maps and institutional affiliations.

Supplementary Information accompanies this paper at (https://doi.org/ 10.1038/s41389-019-0146-y).

Received: 21 December 2018 Revised: 3 May 2019 Accepted: 16 May 2019 Published online: 29 May 2019

\section{References}

1. Omuro, A. \& DeAngelis, L. M. Glioblastoma and other malignant gliomas: a clinical review. JAMA 310, 1842-1850 (2013).

2. Wen, P. Y. \& Kesari, S. Malignant gliomas in adults. N. Engl. J. Med. 359, 492-507 (2008).

3. Cancer Genome Atlas Research N. Comprehensive genomic characterization defines human glioblastoma genes and core pathways Nature $\mathbf{4 5 5}$ 1061-1068 (2008).

4. Phillips, H. S. et al. Molecular subclasses of high-grade glioma predict prognosis, delineate a pattern of disease progression, and resemble stages in neurogenesis. Cancer Cell 9, 157-173 (2006).

5. Verhaak, R. G. et al. Integrated genomic analysis identifies clinically relevant subtypes of glioblastoma characterized by abnormalities in PDGFRA, IDH1, EGFR, and NF1. Cancer Cell 17, 98-110 (2010).

6. Verhaak, R. G. Moving the needle: optimizing classification for glioma. Sci. Transl. Med. 8, 350fs14 (2016).

7. Arimappamagan, A. et al. A fourteen gene GBM prognostic signature identifies association of immune response pathway and mesenchymal subtype with high risk group. PLOS ONE 8, e62042 (2013).
8. Carro, M. S. et al. The transcriptional network for mesenchymal transformation of brain tumours. Nature 463, 318-325 (2010).

9. Lynes, J., Sanchez, V., Dominah, G., Nwankwo, A. \& Nduom, E. Current options and future directions in immune therapy for glioblastoma. Front. Oncol. 8, 578 (2018).

10. Morisse, M. C., Jouannet, S., Dominguez-Villar, M., Sanson, M. \& Idbaih, A. Interactions between tumor-associated macrophages and tumor cells in glioblastoma: unraveling promising targeted therapies. Expert Rev. Neurother. 18, 729-737 (2018).

11. Qian, J. et al. The IFN-gamma/PD-L1 axis between T cells and tumor microenvironment: hints for glioma anti-PD-1/PD-L1 therapy. J. Neuroinflamm. 15, 290 (2018).

12. Lim, M., Xia, Y., Bettegowda, C. \& Weller, M. Current state of immunotherapy for glioblastoma. Nat. Rev. Clin. Oncol. 15, $422-442$ (2018).

13. Chin, C., Lunking, E. S., de la Fuente, M. \& Ayad, N. G. Immunotherapy and epigenetic pathway modulation in glioblastoma multiforme. Front. Oncol. 8, 521 (2018).

14. Hambardzumyan, D., Gutmann, D. H. \& Kettenmann, H. The role of microglia and macrophages in glioma maintenance and progression. Nat. Neurosci. 19, 20-27 (2016).

15. Zanotto-Filho, A. et al. Inflammatory landscape of human brain tumors reveals an NFkappaB dependent cytokine pathway associated with mesenchymal glioblastoma. Cancer Lett. 390, 176-187 (2017).

16. Apte, R. N. et al. Effects of micro-environment- and malignant cell-derived interleukin-1 in carcinogenesis, tumour invasiveness and tumour-host interactions. Eur. J. Cancer 42, 751-759 (2006).

17. Voronov, E. et al. IL-1 is required for tumor invasiveness and angiogenesis. Proc Natl Acad. Sci. USA 100, 2645-2650 (2003).

18. Hayden, M. S. \& Ghosh, S. Shared principles in NF-kappaB signaling. Cell 132, 344-362 (2008).

19. Millet, P., McCall, C. \& Yoza, B. RelB: an outlier in leukocyte biology. J. Leukoc Biol. 94, 941-951 (2013).

20. Lee, D. W. et al. The NF-kappaB RelB protein is an oncogenic driver of mesenchymal glioma. PloS ONE 8, e57489 (2013).

21. Tchoghandjian, A., Jennewein, C., Eckhardt, I., Rajalingam, K. \& Fulda, S. Identification of non-canonical NF-kappaB signaling as a critical mediator of Smac mimetic-stimulated migration and invasion of glioblastoma cells. Cell Death Dis. 4, e564 (2013).

22. Cherry, E. M., Lee, D. W., Jung, J. U. \& Sitcheran, R. Tumor necrosis factor-like weak inducer of apoptosis (TWEAK) promotes glioma cell invasion through induction of NF-kappaB-inducing kinase (NIK) and noncanonical NF-kappaB signaling. Mol. Cancer 14, 9 (2015).

23. Ohtsu, N. et al. Eva1 maintains the stem-like character of glioblastomainitiating cells by activating the noncanonical NF-kappaB signaling pathway. Cancer Res. 76, 171-181 (2016).

24. DiDonato, J. A., Mercurio, F. \& Karin, M. NF-kappaB and the link between inflammation and cancer. Immunol. Rev. 246, 379-400 (2012).

25. Robe, P. A. et al. Early termination of ISRCTN45828668, a phase $1 / 2$ prospective, randomized study of sulfasalazine for the treatment of progressing malignant gliomas in adults. BMC Cancer 9, 372 (2009).

26. Shih, V. F. et al. Control of RelB during dendritic cell activation integrates canonical and noncanonical NF-kappaB pathways. Nat. Immunol. 13, 1162-1170 (2012)

27. Marienfeld, R. et al. RelB forms transcriptionally inactive complexes with RelA p65. J. Biol. Chem. 278, 19852-19860 (2003).

28. Saccani, S., Pantano, S. \& Natoli, G. Modulation of NF-kappaB activity by exchange of dimers. Mol. Cell 11, 1563-1574 (2003).

29. Gupta, A. S., et al. RelB controls adaptive responses of astrocytes during sterile inflammation. Glia https://doi.org/10.1002/glia.23619 (2019).

30. Sasaki, A. et al. Expression of interleukin-1 beta mRNA and protein in human gliomas assessed by RT-PCR and immunohistochemistry. J. Neuropathol. Exp. Neurol. 57, 653-663 (1998).

31. Yamanaka, R., Tanaka, R., Saitoh, T. \& Okoshi, S. Cytokine gene expression on glioma cell lines and specimens. J. Neurooncol.21, 243-247 (1994).

32. Repovic, P. \& Benveniste, E. N. Prostaglandin E2 is a novel inducer of oncostatin-M expression in macrophages and microglia. J. Neurosci. 22, 5334-5343 (2002)

33. Bhardwaj, R. et al. RelB/p50 complexes regulate cytokine-induced YKL-40 expression. J. Immunol. 194, 2862-2870 (2015). 
34. Szulzewsky, F. et al. Human glioblastoma-associated microglia/monocytes express a distinct RNA profile compared to human control and murine samples. Glia 64, 1416-1436 (2016).

35. Liu, T. F. et al. Sequential actions of SIRT1-RELB-SIRT3 coordinate nuclearmitochondrial communication during immunometabolic adaptation to acute inflammation and sepsis. J. Biol. Chem. 290, 396-408 (2015).

36. Chen, X., El Gazzar, M., Yoza, B. K. \& McCall, C. E. The NF-kappaB factor RelB and histone $\mathrm{H3}$ lysine methyltransferase G9a directly interact to generate epigenetic silencing in endotoxin tolerance. J. Biol. Chem. 284, 27857-27865 (2009).

37. Chen, E. Y. et al. Enrichr: interactive and collaborative HTML5 gene list enrichment analysis tool. BMC Bioinforma. 14, 128 (2013).

38. Weintraub, A. S. et al. YY1 is a structural regulator of enhancer-promoter loops. Cell 171, 1573-1588 e28 (2017)

39. Sepulveda, M. A., Emelyanov, A. V. \& Birshtein, B. K. NF-kappa B and Oct-2 synergize to activate the human $3^{\prime}$ Igh hs4 enhancer in B cells. J. Immunol. 172, 1054-1064 (2004).

40. Xia, Y., Shen, S. \& Verma, I. M. NF-kappaB, an active player in human cancers. Cancer Immunol. Res. 2, 823-830 (2014).

41. Jung, J. U. et al. NIK/MAP3K14 regulates mitochondrial dynamics and trafficking to promote cell invasion. Curr. Biol. 26, 3288-3302 (2016).

42. Schaafsma, W. et al. Long-lasting pro-inflammatory suppression of microglia by LPS-preconditioning is mediated by RelB-dependent epigenetic silencing. Brain Behav. Immun. 48, 205-221 (2015).

43. Liu, T. F., Yoza, B. K., El Gazzar, M., Vachharajani, V. T. \& McCall, C. E. NAD +-dependent SIRT1 deacetylase participates in epigenetic reprogramming during endotoxin tolerance. J. Biol. Chem. 286, 9856-9864, (2011).

44. Caito, S. et al. SIRT1 is a redox-sensitive deacetylase that is post-translationally modified by oxidants and carbonyl stress. FASEB J. 24, 3145-3159 (2010).

45. Gao, Z. et al. Sirtuin 1 (SIRT1) protein degradation in response to persistent cJun N-terminal kinase 1 (JNK1) activation contributes to hepatic steatosis in obesity. J. Biol. Chem. 286, 22227-22234 (2011).
46. Petkova, $\mathrm{V}$. et al. Interaction between $\mathrm{Y} Y 1$ and the retinoblastoma protein. Regulation of cell cycle progression in differentiated cells. J. Biol. Chem. 276 7932-7936 (2001)

47. Shi, J., Hao, A., Zhang, Q. \& Sui, G. The role of $\mathrm{YY1}$ in oncogenesis and its potential as a drug target in cancer therapies. Curr. Cancer Drug Targets 15 145-157 (2015).

48. Lu, S. Y., Rodriguez, M. \& Liao, W. S. YY1 represses rat serum amyloid A1 gene transcription and is antagonized by NF-kappa B during acute-phase response. Mol. Cell Biol. 14, 6253-6263 (1994).

49. Siednienko, J. et al. Nuclear factor kappaB subunits RelB and cRel negatively regulate Toll-like receptor 3-mediated beta-interferon production via induction of transcriptional repressor protein YY1. J. Biol. Chem. 286, 44750-44763 (2011).

50. Wei, J., Gabrusiewicz, K. \& Heimberger, A. The controversial role of microglia in malignant gliomas. Clin. Dev. Immunol. 2013, 285246 (2013).

51. Gabrusiewicz, K., et al. Glioblastoma-infiltrated innate immune cells resemble MO macrophage phenotype. JCl Insight 2016;1 https://doi.org/10.1172/jci. insight.85841.

52. Sielska, M. et al. Distinct roles of CSF family cytokines in macrophage infiltration and activation in glioma progression and injury response. J. Pathol. 230, 310-321 (2013).

53. Kordula, T. et al. Oncostatin $M$ and the interleukin- 6 and soluble interleukin-6 receptor complex regulate alpha1-antichymotrypsin expression in human cortical astrocytes. J. Biol. Chem. 273, 4112-4118 (1998).

54. Ma, Y. et al. Ovarian cancer relies on Glucose Transporter 1 to fuel glycolysis and growth: anti-tumor activity of BAY-876. Cancers 2018;11 https://doi.org/ 10.3390/cancers 11010033 .

55. Gao, J. et al. Integrative analysis of complex cancer genomics and clinical profiles using the cBioPortal. Sci. Signal. 6, pl1 (2013).

56. Newman, A. M. et al. Robust enumeration of cell subsets from tissue expression profiles. Nat. Methods 12, 453-457 (2015). 